Vaquero-Solís, M.; Amado Alonso, D.; Sánchez-Oliva, D.; Sánchez-Miguel, P.A.; IglesiasGallego, D. (2020) Emotional Intelligence in Adolescence: Motivation and Physical Activity. Revista Internacional de Medicina y Ciencias de la Actividad Física y el Deporte vol. 20 (77) pp. 119-131 $\mathrm{Http}: / /$ cdeporte.rediris.es/revista/revista77/artinteligencia1127.htm

DOI: $10.15366 /$ rimcafd2020.77.008

\title{
ORIGINAL
}

\section{INTELIGENCIA EMOCIONAL EN LA ADOLESCENCIA: MOTIVACIÓN Y ACTIVIDAD FÍSICA}

\section{EMOTIONAL INTELLIGENCE IN ADOLESCENCE: MOTIVATION AND PHYSICAL ACTIVITY}

\author{
Vaquero-Solís, M. ${ }^{1}$; Amado Alonso, D. ${ }^{2}$; Sánchez-Oliva, D. ${ }^{3}$; Sánchez- \\ Miguel, P.A. ${ }^{1}$ e Iglesias-Gallego, D. ${ }^{1}$ \\ 1 Facultad de Formación del Profesorado. Universidad de Extremadura, Cáceres (España) \\ mivaquero@alumnos.unex.es, pesanchezm@unex.es, diglesia@unex.es \\ ${ }^{2}$ Centro de Estudios del Deporte, Facultad de Ciencias Sociales y Jurídicas, Universidad Rey \\ Juan Carlos, Alcorcón, Madrid (España) diana.amado@urjc.es \\ ${ }^{3}$ Facultad de Ciencias de la Actividad física y del Deporte, Universidad de Extremadura, Cáceres \\ (España) davidsanchez@unex.es
}

Código UNESCO / UNESCO code: 610204 Psicología Escolar / Schoolpsychology.

ClasificacióndelConsejo de Europa / Classification of the Council of Europe: 15 Psicología del Deporte / Sports Psychology.

Recibido 7 de mayo de 2018 Received May 7, 2018

Aceptado 1 de diciembre de 2018 Accepted December 1, 2018

\section{RESUMEN}

El propósito de esta investigación fue analizar las relaciones entre la motivación, actividad física y la inteligencia emocional, y determinar en qué medida incide la actividad física en la inteligencia emocional. Participaron un total de 431 sujetos (12-16 años) de distintos centros educativos. Se valoraron los niveles de autodeterminación, niveles de actividad física y la inteligencia emocional. Los resultados mostraron relaciones significativas de carácter positivo entre la actividad física, los niveles de motivación más autodeterminados y las dimensiones interpersonales, adaptabilidad y estado de ánimo de la inteligencia emocional. Además, los análisis de regresión mostraron que los niveles de actividad física predecían la dimensión interpersonal, adaptabilidad y estado de ánimo de la variable inteligencia emocional. Como conclusión, nuestro estudio destaca la importancia que presenta el estado de ánimo y la adaptabilidad emocional, acompañada de los niveles de motivación más autodeterminados, en la realización de la actividad física. 
PALABRAS CLAVE: Motivación, Actividad Física, Inteligencia Emocional, Adolescentes.

\begin{abstract}
The aim of this research was to examine the relationship between motivation, physical activity and emotional intelligence, and test in which degree physical activity influences on emotional intelligence. A sample size of 431 individuals (12-16 years old) from different schools participated in the study. Levels of selfdetermination, physical activity and emotional intelligence were assessed.

Results showed significant relationships between physical activity, levels of selfdetermination and interpersonal, adaptability and mood state dimension from the emotional intelligence. Moreover, regression analysis revealed that physical activity levels predicted interpersonal, adaptability and mood state dimensions from the emotional intelligence. In conclusion, our study highlights the importance of mood and emotional adaptability, accompanied by more selfdetermined levels of motivation, in the performance of physical activity.
\end{abstract}

KEYWORDS: Motivation, Physical Activity, Emotional Intelligence, Adolescents.

\title{
INTRODUCCIÓN
}

En los últimos años estamos presenciando una tendencia creciente de estudios relacionados con el manejo de las emociones (Al Sudani y Budzynska, 2015; Singh, 2017) en una amplia variedad de contextos como son el educativo (Ferrando et al., 2011), deportivo (Laborde, Dosseville y Allen, 2016), laboral (Njorogey Yazdanifard, 2014), o de la salud (Fernández-Abascal y Martín-Díaz, 2015). En esta línea, el manejo de las emociones ha sido relacionado con multitud de variables psicosociales como son la autoestima (Ruvalcaba-Romero, Fernández-Berrocal,Salazar-Estrada y Gallegos-Guajardo, 2017), los procesos motivacionales (Cera, Almagro, Conde y Sáenz-López, 2015), ylas conductas prosociales (Martin-Raugh, Kell y Motowildo, 2016).

Centrados en el contexto educativo cada vez son más el número de investigaciones que dotan de mayor importancia al cociente emocional en detraimiento del cociente intelectual (Costa y Faria, 2015). En esta línea, el concepto de inteligencia emocional (IE) hace referencia a aquella habilidad centrada en el procesamiento de la información emocional que unifica a las emociones y al razonamiento, permitiendo utilizar dichas emociones en la realización de un razonamiento más efectivo (Mayer y Salovey, 1997). Este concepto engloba procesos individuales como la percepción de las emociones, el uso de las emociones, la regulación de las mismas y el manejo de estas (Mayer, 2001).

En este sentido, el contexto escolar es un medio generador de valores y comportamientos adaptativos. Sin embargo, el centro educativo puede ser un contexto donde emerjan conductas agresivas y antisociales (Del Rey y Ortega, 
2008; Smith, 2004). Por ello, es importante el trabajo de la IE en el contexto educativo ya que son múltiples los beneficios obtenidos de ella, como son la mejora de la salud física y mental (Fernández-Berrocal y Extremera, 2016; Zamarripa, Castillo, Tomas, Tristan y Álvarez, 2016), un mayor bienestar personal y social (Castillo, Almagro, García y Buñuel, 2015), aumento de las conductas prosociales (Romero, Guajardo y Nava2017) y un mejor rendimiento académico (Ferrando et al, 2011).

Este estudio ha sido desarrollado desde dos marcos conceptuales que se han basado en los beneficios que reporta la actividad física como nexo de unión de ambos: por un lado, se pretende trabajar la IE bajo el modelo de Bar-On (2000), donde las competencias y habilidades pertenecientes a la IE son agrupadas en cinco habilidades sociales y emocionales principales: habilidades interpersonales, habilidades intrapersonales, adaptabilidad, manejo del estrés y estado de ánimo. En este sentido, importantes investigaciones sugieren que el termino IE está asociado al futuro éxito en la vida (Goleman, 2000; Salovey y Mayer, 1990; Joseph y Newman, 2010), a la salud mental y física (Ader, 2001) y a los comportamientos adaptativos y habilidades sociales (Mayer, Salovey y Caruso, 2008).También, el termino IE ha sido relacionado en ciertas ocasiones con la actividad física (Al Sudani y Budzynska, 2015; Bhullar, Schutte y Malouff, 2013; Cechini, Méndez-Giménez y García Romero, 2018; Ladino, GonzálezCorrea, González-Correa y Caicedo,2016; Li, Lu y Wang, 2009; Singh, 2017; Zysberg y Hemmel, 2017), Mejorando el estado de ánimo y el manejo del estrés, (Newman, Joseph, MacCann, 2010). Asimismo, no son muchos los investigadores que han tratado la importancia de esta sobre el estado emocional (Saleem y Mahmood, 2013; Singh, 2017). No obstante, estudios previos señalan que la realización de actividad física contribuye al desarrollo de la autoestima, el autoconcepto y la autoconfianza (Biddle y Asare, 2011), así como la sensación de bienestar, disminución de la ansiedad y una mejora de la comunicación y empatía (De Benito y Lujan, 2013; Ros, Moya-Faz y Garcés de los Fayos, 2013).

Por otro lado, otros estudios también han relacionado a la IE con la motivación (Perreault, Mask, Morgan, y Blanchard, 2014; Petrides, 2010), mostrando en sus estudios la importancia de la motivación autodeterminada en el manejo de las emociones. En esta línea, Goleman (1995) la señala como uno de los principios básicos que componen la inteligencia emocional. Teniendo en cuenta lo anterior, esta investigación también se ha contextualizado bajo los principios de la teoría de la autodeterminación (Deci y Ryan, 2000), una macro-teoría de la personalidad y la que propone cómo el contexto puede influir en las razones para hacer determinadas actividades. Esta teoría se centra en la medida en que los comportamientos son voluntarios o autodeterminados, sosteniendo que la motivación es un continuo de autodeterminación, diferenciado entre motivación autónoma (regulación intrínseca e identificada), motivación controlada (regulaciones introyectada y externa) y desmotivación. La regulación intrínseca constituye el nivel más alto de motivación, en la cual el desarrollo de la actividad en sí constituye el objetivo y la gratificación, suscitando también sensaciones de competencia y autorrealización. La regulación identificada se refiere a la involucración en una determinada actividad por la valoración positiva que se realiza de la misma. Dentro de la motivación controlada se sitúa la regulación introyectada, asociada a personas que realizan una actividad para evitar 
sentimientos de culpabilidad con el objetivo de mejorar el ego personal o el orgullo. Seguidamente, se sitúa la regulación externa que hace referencia a la realización de una actividad para conseguir una recompensa externa, o evitar un castigo. Finalmente, se encuentra la desmotivación, que representa la ausencia de motivación tanto intrínseca como extrínseca (Deci y Ryan, 2000).

Así pues, son numerosos los estudios que abordan la influencia de la motivación sobre el nivel de actividad física (Murcia, Silva, Pardo, y Hernández, 2016; Ruiz, Ortiz-Camacho, García-Montes, Baena-Extremera y Baños, 2018;SolomonMoore, Sebire, Thompson, Zahra, Lawlor y Jago, 2017;Texeira, Carraca, Markland, Silva y Ryan, 2012). Sin embargo, no es tan amplia la literatura disponible sobre actividad física e IE en el ámbito educativo (Al Sudani y Budzynska, 2015; Ferrándiz, Hernández, Bermejo, Ferrando y Sainz, 2012; Ladino et al., 2016; Li, Lu y Wang, 2009,), y mucho menos son los estudios que trabajan conjuntamente el nivel motivacional, la actividad física y IE (Cera, Almagro, Conde y Sáenz-López, 2015; De Benito y Lujan, 2013).

El propósito de nuestra investigación ha sido analizar las relaciones que se producen entre los tipos de motivación, los niveles de actividad física y la IE. Más específicamente el objetivo de nuestra investigación fue conocer en qué medida la motivación hacia la actividad física predecía los niveles de actividad física, y está a su vez a la IE.

Se establecieron las siguientes hipótesis:

$\mathrm{H}_{0}$ - Los niveles de motivación más autodeterminados estarán relacionados con una mayor práctica de actividad física y se relacionaran de forma positiva con los niveles de inteligencia emocional.

$\mathrm{H}_{1}$ - Aquellos tipos de motivación menos autodeterminados estarán relacionados con una menor práctica de actividad física y se relacionaran de forma negativa con los niveles de inteligencia emocional.

$\mathrm{H}_{2}$-Los niveles de motivación más autodeterminados predecirán una mayor práctica de actividad física, y ésta repercutirá en una mayor IE.

\section{MÉTODO}

\section{Participantes}

Un total de 431 estudiantes españoles de Educación Secundaria Obligatoria (ESO) con edades comprendidas entre 12 y 16 años $(M=13,54 ; D T=0,99), 209$ $(48,5 \%)$ para el género masculino y $222(51,5 \%)$ para el género femenino, participaron en esta investigación. Pertenecían a diferentes escuelas de la Comunidad Autónoma de Extremadura (España).La muestra fue seleccionada a través de un muestreo de selección intencionada por conglomerados teniendo en cuenta la disponibilidad del investigador y la zona geográfica.

Instrumentos 
Tipo de motivación hacia la actividad física. Se utilizó la versión en castellano (Moreno, Cervelló y Martínez, 2007) del Cuestionario de Regulación de la Conducta en el Ejercicio Físico-2 (BREQ-2; Markland y Tobin, 2004). Dicho cuestionario está compuesto por 19 ítems agrupados en 5 factores, los cuales empiezan con la frase inicial "Yo hago ejercicio...". Los factores hacen referencia a la regulación intrínseca (4 ítems, eg. "Porque creo que el ejercicio es divertido"; $\alpha=0,84$ ), regulación identificada (4 ítems, eg. "Porque valoro los beneficios que tiene el ejercicio físico"; $\alpha=0,69$ ) regulación introyectada ( 3 ítems, eg. "Porque me siento culpable sino lo práctico"; $\alpha=0,70$ ), regulación externa (4 ítems, eg. "Porque los demás me dicen que debo hacerlo"; $\alpha=0,70)$ y desmotivación (4 ítems, eg. "No veo porque tengo que hacerlo"; $\alpha=0,72$ ). Las respuestas al cuestionario se realizaban en una escala Likert que varía de 1 a 5 , donde el 1 corresponde a nada verdadero y el 5 a totalmente verdadero.

Niveles de actividad física. La actividad física fue analizada a través del Cuestionario de Actividad Física para adolescentes (Physical Activity Questionary for Adolescents: PAQ-A) (Kowalsky, Crocker y Kowalski, 2004). Dicho cuestionario está compuesto por 9 ítems que valoran el nivel de actividad física que el adolescente realizó en los últimos 7 días, mediante una escala Likert de 5 puntos: durante su tiempo libre, durante las clases de educación física, así como en diferentes horarios durante los días de clase (comida, tardes y noches) y durante el fin de semana. El resultado es una puntuación de 1 a 5 que permite establecer una graduación en el nivel de actividad física (Martínez-Gómez et al., 2009). Su puntuación final se obtiene a partir de la media aritmética de 8 de los 9 ítems, pues el último ítem valora si el participante estuvo enfermo durante la última semana (Martínez-Gómez et al., 2009). Finalmente, el coeficiente alfa de Cronbach obtenido para la presente muestra fue de $(\alpha=0,79)$.

Inteligencia emocional. Para la evaluación de la IE en adolescentes se ha empleado el Inventario para el Cociente Emocional en Jóvenes (Emotional Quotient inventory: Young Version: EQ-i: YV) (Bar-On y Parker, 2000), y validado al castellano por Ferrándiz, Hernández, Bermejo, Ferrando y Sainz (2012). Esta versión está compuesta por 54 ítems divididos en cinco dimensiones. Los análisis de fiabilidad revelaron los alfas de Cronbach para cada una de las dimensiones: intrapersonal ( 6 ítems, eg. "Me resulta fácil decirle a la gente como me siento"; $\alpha=0,70$ ), interpersonal (12 ítems, eg. "Entiendo bien como se sienten otras personas"; $\alpha=0,73$ ), adaptabilidad (10 ítems, eg. "Es fácil para mí atender a cosas nuevas"; $\alpha=0,80$ ), manejo del estrés (12 ítems, eg. "Puedo estar tranquilo cuando estoy enfadado"; $\alpha=0,70$ ) y estado de ánimo general (14 ítems, eg. "Soy feliz"; $\alpha=0,85)$. Además, esta escala añade seis ítems creados por el autor para medir el grado en que los sujetos responden al azar o distorsionan sus respuestas en función del efecto de deseabilidad social. Las respuestas al cuestionario se realizaban con una escala tipo Likert de 4 puntos, donde el 1 era muy rara vez y el 4 muy a menudo.

Procedimiento 
La elaboración de este estudio se ha llevado a cabo en varias fases. En primer lugar, nos pusimos en contacto con los centros educativos colaboradores, y se explicó que el cuestionario era totalmente anónimo, por lo que no se comprometía la identidad de los participantes. En lo pertinente a las normas éticas, el estudio recibió previamente la aprobación del comité ético de la Universidad de Extremadura. Además, todos los participantes fueron tratados bajo los principios éticos y código de conducta de la American PsychologicalAssociation(2002) para este tipo de investigaciones. Se solicito permiso a profesores, padres y madres, y se les explico en qué consistiría el estudio y cuales serian las variables evaluadas. El procedimiento llevado a cabo por parte del investigador era presentarse, explicar brevemente de lo que trataría el cuestionario y dejar claro que no era una prueba de evaluación para que los alumnos fueran lo más sinceros posible. El tiempo aproximado para la cumplimentación del cuestionario fue de 25 minutos.

\section{Análisis estadísticos}

Para la realización del análisis de datos se utilizó el paquete estadístico SPSS 23.0, con el cual se realizaron diferentes pruebas para determinar la naturaleza de los datos, la pruebakolmogorov-Smirnof para muestras independientes, la prueba aleatoria Rachas, la prueba de homocedasticidad o igualdad entre varianzas de Levene y la naturaleza de los datos paramétricos. Posteriormente, se analizaron los estadísticos descriptivos y las correlaciones bivariadas para todas las variables del estudio con el fin de analizar la significatividad de las relaciones entre las variables. Finalmente, se realizaron regresiones lineales con el fin de comprobar la capacidad predictiva de una variable sobre otra.

Estadísticos descriptivos y análisis de correlación.

En la tabla 1, se muestran los estadísticos descriptivos y correlaciones bivariadas. En líneas generales, las dimensiones estado de ánimo ( $M=4,09 ; D T$ $=0,55)$ y dimensión interpersonal $(M=4,06 ; D T=0,46)$, pertenecientes a la IE, presentaron valores más elevados que el resto de dimensiones de la misma. Asimismo, la regulación intrínseca $(M=4,00 ; D T=0,93)$ e identificada $(M=3,55$; $D T=0,77)$ presentaron las puntuaciones medias más altas en los tipos de motivación.

Además, el análisis de correlaciones mostró asociaciones positivas y significativas entre la actividad física y la dimensión interpersonal $(r=0,19$; $p<0,01)$, adaptabilidad $(r=0,21 ; p<0,01)$, y estado de ánimo de la IE $(r=0,23$; $p<0,01$ ). Asimismo, existe una fuerte correlación entre la actividad física y las dimensiones más autodeterminadas de la motivación $(p<0,01)$. Por el contrario, la regulación externa se asoció significativamente de manera negativa con la actividad física $(r=-0,11 ; p<0,01)$, la adaptabilidad $(r=-0,11 ; p<0,01)$, y las regulaciones intrínsecas $(r=-0,27 ; p<0,01)$, e identificadas $(r=-0,16)$ de la motivación. En relación a la desmotivación, se relacionó de manera significativamente negativa con las variables actividad física, interpersonal, adaptabilidad y las regulaciones intrínsecas, identificadas $(p<0,01)$ e introyectada $(p<0,05)$ de la motivación, mientras que se relacionó 
significativamente de forma positiva con el manejo del estrés $(p<0,05)$ y la regulación externa $(p<0,01)$.

Tabla 1.Estadísticos descriptivos y Análisis de correlación de las variables de estudio

\begin{tabular}{|c|c|c|c|c|c|c|c|c|c|c|c|}
\hline VARIABLES & 1 & 2 & 3 & 4 & 5 & 6 & 7 & 8 & 9 & 10 & 11 \\
\hline 1.PAQ-A & - & 0,02 & $0,19^{* *}$ & $-0,05$ & $0,21^{* *}$ & $0,23^{* \star}$ & $0,42^{\star \star}$ & $0,40^{* *}$ & $0,22^{\star \star}$ & $-0,11^{*}$ & $-0,22^{\star \star}$ \\
\hline 2.Intra & - & - & $0,32^{* *}$ & 0,01 & $0,23^{* *}$ & $0,32^{* *}$ & $0,10^{*}$ & $0,11^{*}$ & $0,13^{* *}$ & 0,05 & 0,01 \\
\hline 3.Inter & - & - & - & 0,04 & $0,32^{* *}$ & 0,30 ** & $0,21^{* *}$ & $0,32^{\star *}$ & $0,15^{\star *}$ & $-0,06$ & $-0,21^{* *}$ \\
\hline 4.M. Estrés & - & - & - & - & 0,02 & $-0,11^{*}$ & $0,14^{\star *}$ & $-0,10^{*}$ & 0,06 & 0,04 & $0,12^{*}$ \\
\hline 5. Adaptabilidad & - & - & - & - & - & $0,36^{\star *}$ & $0,23^{* *}$ & $0,26^{\star *}$ & $0,09^{*}$ & $-0,11^{*}$ & $-0,15^{\star *}$ \\
\hline 6.E.Ánimo & - & - & - & - & - & - & $0,30^{* *}$ & $0,33^{* *}$ & 0,06 & $-0,07$ & $-0,07$ \\
\hline 7.R. Intrínseca & - & - & - & - & - & - & - & $0,63^{* *}$ & $0,12^{*}$ & $-0,28^{* *}$ & $-0,48^{* *}$ \\
\hline 8. R. Identificada & - & - & - & - & - & - & - & - & $0,34^{\star *}$ & $-0,17^{\star *}$ & $-0,43^{\star *}$ \\
\hline 9. R. Introyectada & - & - & - & - & - & - & - & - & - & $0,24^{\star *}$ & -0.03 \\
\hline 10.R.Externa & - & - & - & - & - & - & - & - & - & - & $0,43^{\star *}$ \\
\hline 11.Desmotivación & - & - & - & - & - & - & - & - & - & - & - \\
\hline 12. $M$ & 2,79 & 2,85 & 4,06 & 2,57 & 3,51 & 4,09 & 4,00 & 3,55 & 2,53 & 1,74 & 1,62 \\
\hline 13. $D T$ & 0,67 & 0,83 & 0,46 & 0,55 & 0,60 & 0,55 & 0,93 & 0,77 & 1,06 & 0,79 & 0,76 \\
\hline 14. $\alpha$ & 0,79 & 0,70 & 0,73 & 0,70 & 0,80 & 0,85 & 0,84 & 0,69 & 0,70 & 0,70 & 0,72 \\
\hline
\end{tabular}

Análisis de regresión

En la tabla 2 se exponen los resultados del análisis de regresión lineal realizado, a través del método introducir, incluyendo la actividad física como variable dependiente y los tipos de motivación como predictores. El modelo explicó un $23,7 \%$ del total de la varianza, donde las regulaciones intrínseca $(b=0,23 ; p<$ $0,01)$, identificada $(b=0,10 ; p<0,01)$ e introyectada $(b=0,10 ; p<0,05)$ predijeron positiva y significativamente la $A F(p<0,05)$, mientras que la regulación externa lo hizo negativamente $(p<0,05)$. Asimismo, el análisis de regresión viene explicado por la siguiente ecuación $\left(Y=1,316+0,036 x_{1}+\right.$ $\left.0,012 x_{2}+0,1023+0,102 x_{4}+0,229 x_{5}\right)$, donde $Y$ es la constante $A F, y\left(X_{1}, X_{2}, X_{3}\right.$, $\mathrm{X}_{4}, \mathrm{X}_{5}$ ) son el resto de variables predictoras del modelo.

Tabla 2.Análisis de regresión lineal Motivación-Actividad Física

\begin{tabular}{lccccc}
\hline & \multicolumn{5}{c}{ Actividad Física } \\
\cline { 2 - 6 } Variables & $B$ & $R^{2}$ & $\beta$ & $T$ & $P$ \\
\hline & 0,23 & 0,24 & & \\
1.R. Intrínseca & 0,23 & $0,32^{* *}$ & 4,36 & 0,00 \\
2.R. Identificada & 0,10 & & $0,13^{*}$ & 3,59 & 0,03 \\
3.R. Introjectada & 0,10 & $0,16^{*}$ & 2,03 & 0,01 \\
4.R. Externa & $-0,12$ & $-0,01$ & $-2,34$ & 0,28 \\
5. Desmotivación & 0,03 & 0,04 & 0,53 & 0,59 \\
\hline & \multicolumn{5}{c}{$0,0,01,{ }^{*} p<0,05$}
\end{tabular}

En el siguiente análisis de regresión (tabla 3), se incluyó como variable dependiente cada una de las variables de la IE, y como predictor los niveles de actividad física. Los resultados de los análisis de regresión llevados a cabo para 
las dimensiones estado de ánimo ( $\mathrm{b}=0,19 ; p<0,01)$, adaptabilidad $(\mathrm{b}=0,19 ; p$ $<0,01)$ e interpersonal ( $b=0,12 ; p<0,01)$ mostraron valores positivos y significativos $(p<0,05)$ y explicaron $5 \%, 4,3 \%$ y $3,2 \%$ del total de la varianza, respectivamente. Por otro lado, los resultados de los análisis de regresión para las dimensiones manejo del estrés e intrapersonal ofrecieron valores no significativos $p>0,05 y$ explicaron un $0,1 \%$ y un $0,3 \%$ respectivamente del total de la varianza.

Tabla 3. Análisis de regresión lineal Actividad Física-Inteligencia Emocional

\begin{tabular}{|c|c|c|c|c|c|}
\hline \multicolumn{6}{|c|}{ Estado de Animo } \\
\hline Variables & $B$ & $R^{2}$ & $\beta$ & $T$ & $P$ \\
\hline 1.A.Física & 0,19 & 0,50 & $0,23^{* *}$ & 4,77 & 0,00 \\
\hline \multicolumn{6}{|c|}{ Manejo del estrés } \\
\hline Variables & $B$ & $R^{2}$ & $\beta$ & $T$ & $P$ \\
\hline 1.A.Física & $-0,05$ & 0,01 & $-0,06$ & $-1,26$ & 0,21 \\
\hline \multicolumn{6}{|c|}{ Adaptabilidad } \\
\hline Variables & $B$ & $R^{2}$ & $\beta$ & $T$ & $P$ \\
\hline 1.A.Física & 0,19 & 0,43 & $0,21^{* *}$ & 4,41 & 0,00 \\
\hline \multicolumn{6}{|c|}{ Interpersonal } \\
\hline Variables & $B$ & $R^{2}$ & $\beta$ & $T$ & $P$ \\
\hline 1.A.Física & 0,12 & 0,32 & $0,18^{* *}$ & 3,79 & 0,00 \\
\hline \multicolumn{6}{|c|}{ Intrapersonal } \\
\hline Variables & $B$ & $R^{2}$ & $\beta$ & $T$ & $P$ \\
\hline 1.A.Física & 0,09 & 0,03 & 0,08 & 1,55 & 0,12 \\
\hline
\end{tabular}

\section{DISCUSIÓN}

Basándonos en el marco teórico que supone la Teoría de la Autodeterminación (Deci y Ryan, 2000) y el modelo de IE propuesto por Bar-On (2000), nuestro principal objetivo ha sido analizar las relaciones que se producen entre la motivación, la actividad física y la IE. Por ello, en relación a la primera hipótesis, se postuló que los niveles de motivación más autodeterminados estarían relacionados con una mayor práctica de actividad física y se relacionarán de forma positiva con la inteligencia emocional. En esta línea, nuestros hallazgos mostraron una mayor relación entre los niveles más autodeterminados y la actividad física. Estos resultados, son congruente con los encontrados por (Cera, Almagro, Conde y Sáenz-López, 2015) que confirman la importancia que presenta la motivación autodeterminada en la práctica de AF. En este sentido, aquellos alumnos que realicen actividad física solo por el hecho de disfrute y gratificación presentaran una motivación más autodeterminada (Deci y Ryan, 2000).

En referencia, a la relación de carácter positiva producidas entre los niveles de motivación, actividad física e inteligencia emocional, los resultados son congruentes con los hallados en estudios previos como el de Benito y Lujan (2013), que confirman la relación existente entre los niveles de motivación y la IE y añade que los niveles de IE fueron mayores para los practicantes de deporte que para aquellos que solo realizaban educación física. 
Este hecho puede explicarse a través de la satisfacción de las necesidades psicológicas básicas (Deci y Ryan, 2000), donde la satisfacción de las relaciones sociales puede estar estrechamente relacionada con las dimensiones intrapersonal e interpersonal del modelo de (Bar-On, 2000). Así pues, un alumno que se relacione de forma correcta con sus compañeros, poseerá una mayor IE y tendrá una actitud más receptiva ante la realización de actividad física (Cera, Almagro, Conde y Sáenz-López, 2015). De igual modo, Sánchez -Gutiérrez y Araya (2014) confirman la relación existente entre los niveles de actividad física e IE, donde fueron mayores los niveles de IE en aquellas personas que mostraron más nivel de actividad física. Sin embargo, Ladino, et al., (2016) en su estudio mostró que la educación física produjo cambios en la IE, pero estos cambios no llegaron a ser significativos.

Por otro lado, en referencia a la segunda hipótesis, se enunció que los niveles más bajos de motivación estarían relacionados con una menor práctica de actividad física y menores niveles de IE. En este sentido, nuestro estudio mostró relaciones negativas y significativas entre la actividad física y la regulación externa. En relación a este hecho Murcia et al. (2016), mostraron que algunos motivos externos, como motivos sociales y la imagen o apariencia física, están relacionadas con un alta práctica físico-deportiva.

En relación a la motivación extrínseca los y niveles inferiores de IE, nuestros resultados no mostraron significación a excepción de la dimensión adaptabilidad. Estudios previos señalan que los niveles de motivación extrínsecos están relacionados con aquellas conductas más desadaptativas (Zamarripa et al., 2016).

Concerniente a la tercera hipótesis, donde se postuló una serie de predicciones producidas entre los niveles de motivación, los cuales, predecirían a una mayor práctica de actividad física, y está, repercutirá en una mayor IE. Nuestros resultados mostraron por un lado, que un fuerte papel de los niveles de motivación más autodeterminados actúan como predictores del nivel de actividad física. Estos resultados son consistentes con los hallazgos encontrados previamente (Ruiz et al., 2018; Solomon-Moore et al., 2017; Texeira et al., 2012), donde queda ampliamente demostrado que los niveles de motivación predicen a la actividad física.

Por otro lado, respecto al papel de la actividad física como predictor de los niveles de IE, los resultados mostraron valores significativos para las dimensiones estado de ánimo, interpersonal y adaptabilidad. Estos datos ponen de manifiesto que los adolescentes que realizan una mayor práctica de actividad física reflejan mayores puntuaciones para las dimensiones nombradas anteriormente. Estudios previos (Al Sudani y Budzynska, 2015; Singh, 2017), indican que altos niveles de actividad física tienen un incremento en el nivel de IE. De igual modo, en referencia a lasdimensiones adaptabilidad, estado de ánimo e interpersonal, Zysberg y Hemmel (2017) exponen que el manejo de las emociones y el componente interpersonal está asociado con la actividad física. En esta línea, Castillo, Almagro, García y Buñuel (2015) exponen que la actividad física puede asociarse mejor con ciertos aspectos de la inteligencia emocional y 
menos con otros. Además, en contraposición a nuestros resultados, un estudio de índole similar (Li et al., 2009) concluyó que la educación física puede que no sea el determinante de la IE, sino que, más bien al contrario, la IE prediga los niveles de actividad física.

\section{CONCLUSIÓN}

De acuerdo con los resultados obtenidos, nuestro estudio ha hecho énfasis en la importancia que tiene el nivel motivacional sobre el mantenimiento de los niveles de actividad física, y la repercusión de estos sobre la IE. En este sentido, este estudio presenta algunas limitaciones como es la naturaleza transversal de nuestra investigación, la cual no ha permitido establecer relaciones de causaefecto. Además, es importante destacar la dificultad que puede ocasionar en la compresión de los alumnos términos del instrumento que valoró la IE. A pesar de estas limitaciones, nuestros resultados nos han llevado a concluir en la importancia que presentan los niveles de actividad física sobre alguna de las dimensiones que engloban a la inteligencia emocional, como son la adaptabilidad, el estado de ánimo, y la inteligencia interpersonal, dimensiones que tienen una alta presencia en el contexto de la actividad físico-deportiva, y favorecen en la proliferación de relaciones sociales y en la adquisición de un buen estado de ánimo. Por ello, consideramos necesario el desarrollo de más estudios y aplicaciones de intervención que tengan en cuenta otras variables estrechamente relacionada con la inteligencia emocional, como pueden ser las necesidades psicológicas básicas, el bienestar y los contextos donde se desarrolla la actividad física.

\section{REFERENCIAS BIBLIOGRAFICAS}

Ader, R. (2001). Psychoneuroimmunology. Current Directions in Psychological Science, 10(3), 94-98.

Al Sudani, A. A. D. y Budzynska, K. (2015). Emotional intelligence, physical activity and coping with stress in adolescents. International Journal of Science Culture and Sport, 3(2), 98-104. doi:10.5281/zenodo.1001774

Bar-On, R. y Parker, J.D.A. (2000). The Bar-On Emotional Quotient Inventory: Youth Version (EQ-i:YV). Technical Manual (translated to Spanish by $C$. M. Caraballo y O. Villegas). Toronto, Canada: Multi-Health Systems, Inc.

Bhullar, N., Schutte, N. S. y Malouff, J. M. (2013). The nature of well-being: The roles of hedonic and eudaimonic processes and trait emotional intelligence. The Journal of Psychology, 147(1), 1-16. doi:10.1080/00223980.2012.667016

Biddle, S.J. y Asare, M. (2011). Physical activity and mental health in children and adolescents: A review of reviews. British Journal of Sports Medicine, 45(11), 886-895. doi:10.1136/bjsports-2011-090185

Cechini, J .A., Méndez-Giménez, A., y García Romero, C (2018). Validación del cuestionario de inteligencia emocional en educación física. Revista de Psicología del Deporte, 27(1), 87-96.

Cera, E., Almagro, B. J., Conde, C. y Sáenz-López, P. (2015). Inteligencia emocional y motivación en educación física en secundaria. RETOS. Nuevas Tendencias en Educación Física, Deporte y Recreación, 27, 8-13. 
Conde, C. y Almagro, B. J. (2013). Estrategias para desarrollar la inteligencia emocional y la motivación en el alumnado de Educación Física. E-motion. Revista de Educación, Motricidad e Investigación, (1), 212-220.

De Benito, M. M. y Luján, J. I. G. (2013). Inteligencia emocional, motivación autodeterminada y satisfacción de necesidades básicas en el deporte. Cuadernos de Psicología del Deporte, 12(2), 39-44.

Deci, E. L. y Ryan, R. M. (2000). The "what" and "why" of goal pursuits: Human needs and the selfdetermination of behaviour. Psychological Inquiry, 11, 227-268.

Fernández-Berrocal, P. y Extremera, N. (2016). Ability emotional intelligence, depression, and well-being. Emotion Review, 8(4), 311-315.doi: $10.1177 / 1754073916650494$

Ferrándiz, C., Hernández, D., Bermejo, R., Ferrando, M. y Sáinz, M. (2012). La inteligencia emocional y social en la niñez y adolescencia: validación castellana de un instrumento para su medida. Revista de Psicodidáctica, 17(2), 309-339. doi: 10.1387/Rev.Psicodidact.2814

Ferrando, M., Prieto, M. D., Almeida, L. S., Ferrandiz, C., Bermejo, R., LopezPina, J. A., et al. (2011). Trait Emotional Intelligence and Academic Performance: Controlling for the Effects of $\mathrm{IQ}$, Personality, and SelfConcept. Journal of Psychoeducational Assessment, 29(2), 150-159.doi: 10.1177/0734282910374707

Goleman, D. (1995). Emotional Intelligence. New York: Bantam Books

Goleman, D. (2000). Estilos de liderazgo. Harvard Business Review.

Kowalski, K. C., Crocker, P. R. y Donen, R. M. (2004). The physical activity questionnaire for older children (PAQ-C) and adolescents (PAQ-A) manual. College of Kinesiology, University of Saskatchewan, 87(1), 1-38.

Ladino, P. K., González-Correa, C. H., González-Correa, C. A. y Caicedo, J. C. (2016). Ejercicio físico e inteligencia emocional en un grupo de estudiantes universitarias. Revista Iberoamericana de Psicología del Ejercicio y el Deporte, 11(1), 31-36.

Li, G. S. F., Lu, F. J. y Wang, A. H. H. (2009). Exploring the relationships of physical activity, emotional intelligence and health in Taiwan college students. Journal of Exercise Science \& Fitness, 7(1), 55-63.

Markland, D. y Tobin, V. (2004). A modification to Behavioural Regulation in Exercise Questionnaire to include an assement of amotivation. Journal of Sport and Exercise Psychology, 26(2), 191-196. doi: 10.1123/jsep.26.2.191

Martínez-Gómez, D., Martínez-de-Haro, V., Pozo, T., Welk, G. J., Villagra, A., Calle, M. E y Veiga, O. L. (2009). Fiabilidad y validez del cuestionario de actividad física PAQ-A en adolescentes españoles. Revista Española de Salud Pública, 83(3), 427-439.

Mayer, J. D. (2001). Emotion, Intelligence and Emotional Intelligence. Handbook of affect and social cognition. J.P. Forgas.

Mayer, J. D., y Salovey, P. (1997). What is emotional intelligence? In P. Salovey \& D. J. Sluyter (Eds.), Emotional development and emotional intelligence: Educational implications (pp. 3-34). New York: Harper Collins.

Mayer, J. D., Salovey, P., y Caruso, D. R. (2008). Emotional intelligence: new ability or eclectic traits? American Psychologist, 63, 503-517. doi:10.1037/0003-055x.63.6.503 
Méndez-Giménez, A., Cecchini, J. A. y Fernández-Río, J. (2016). Pasión por el deporte, actividad física vigorosa y satisfacción con la vida. Revista de Psicología del Deporte, 25(1), 73-79.

Moreno, J.A., Cervelló, E. y Martínez, A. (2007). Measuring self-determination motivation in a physical fitness setting; validation of the Behavioral Regulation in Exercise Questionnaire-2 (BREQ-2) in Spanish sample. The Journal of Sports Medicine and Physical Fitness, 47(3), 366-378.

Murcia, J. A., Silva, F. B., Pardo, P. J., Sierra, A. C. y Hernández, E. H. (2016). Motivación, frecuencia y tipo de actividad en practicantes de ejercicio físico/Motivation, frequency and activity type in physical exercise practitioners. Revista Internacional de Medicina y Ciencias de la Actividad Física y del Deporte, 12(48), 649-662.

Newman DA, Joseph DL, MacCann C. (2010) Emotional intelligence and job performance: the importance of emotion regulation and emotional labor context Industrial and Organizational Psychology, 3(2),159164.doi:doi.org/10.1111/j.1754-9434.2010.01218.x

Petrides KV. (2010). Trait emotional intelligence theory. Industrial and Organizational Psychology. 3(2),136-139.doi: 10.1111/j.17549434.2010.01213.x

Romero, N. A. R., Guajardo, J. G., y Nava, J. M. F. (2017). Competencias socioemocionales como predictoras de conductas prosociales y clima escolar positivo en adolescentes. Revista interuniversitaria de formación del profesorado, (88), 77-90.

Ros, A., Moya, F. y Garcés de Los Fayos, E. (2013). Inteligencia emocional y deporte: situación actual del estado de la investigación. Cuadernos de Psicología del Deporte, 13(1), 105-112.

Ruiz-Juan, F., Ortiz-Camacho, M. M., García-Montes, M. E., Baena-Extremera, A., y Baños, R. (2018). Predicción transcultural del clima motivacional en educación física. Revista Internacional de Medicina y Ciencias de la Actividad Física y del Deporte, 18 (69) pp. 165183.doi:10.15366/rimcafd2018.69.01

Sánchez-Gutiérrez, G. y Araya-Vargas, G. (2014). Atención plena, inteligencia emocional, género, área de estudio y reporte de ejercicio en estudiantes universitarios costarricenses. Revista Iberoamericana de Psicología del Ejercicio y el Deporte, 9(1), 19-36.

Saleem, S. y Mahmood, Z. (2013). Mental health problems in university students: A prevalenve study. FWU Journal of Social Sciences, 7(2), 124-130

Singh, H. (2017). Relationship between leisure-time physical activity and emotional intelligence in female university students: A correlational. European Journal of Physical Education and Sport Science, 3 (10), 209-216. doi:10.5281/zenodo.1001774.

Solomon-Moore, E., Sebire, S., Thompson, JL., Zahra, J., Lawlor, DA. Y Jago, R. (2017). Are parents' motivations to exercise and intention to engage in regular family-based activity associated with both adult and child physical activity? BMJ Open Sport \& Exercise Medicine, 2(1), e000137. doi:10.1136/bmjsem-2016-000137.

Teixeira, P. J., Carraça, E. V, Markland, D., Silva, M. N., y Ryan, R. M. (2012). Exercise, physical activity, and self-determination theory: A systematic 
review. International Journal of Behavioral Nutrition and Physical Activity, 9(1), 78-107. doi:10.1186/1479-5868-9-78

Zysberg, L. y Hemmel, R. (2017). Emotional Intelligence and Physical Activity. Journal of Physical Activity and Health, 15 (1), 53-56. doi:10.1123/jpah.2016-0654

Zamarripa, J., Castillo, I., Tomás, I., Tristán, J., y Álvarez, O. (2016). El papel del profesor en la motivación y la salud mental de los estudiantes de educación física. Salud mental, 39(4), 221-227. doi:10.17711/SM.01853325.2016 .026

Total references / Referencias totales: 38 (100\%)

Journal'sownreferences / Referencias propias de la revista: 2 (5.26)

Rev.int.med.cienc.act.fís.deporte- vol. 20 - número 77 - ISSN: 1577-0354 